\title{
Longitudinal pathways of exclusive and polytobacco cigar use among youth, young adults and adults in the USA: findings from the PATH Study Waves 1-3 (2013-2016)
}

\author{
Kathryn C Edwards (ㅇ, ', Eva Sharma (1) , ${ }^{1}$ Michael J Halenar (10), \\ Kristie A Taylor (ㅇ, , Karin A Kasza (1), ${ }^{2}$ Hannah Day, ${ }^{3}$ Hoda T Hammad, ${ }^{3}$ \\ Gabriella Anic, ${ }^{3}$ Maansi Bansal-Travers (10), ${ }^{2}$ Jean Limpert, ${ }^{3}$ Lisa D Gardner (10 , ${ }^{3}$ \\ Nicolette Borek, ${ }^{3}$ Heather L Kimmel $\odot 1,{ }^{4}$ Wilson M Compton, ${ }^{4}$ Andrew Hyland, ${ }^{2}$ \\ Cassandra A Stanton (1) 1,5
}

\begin{abstract}
- Additional material is published online only. To view please visit the journal online (http://dx.doi.org/10.1136/ tobaccocontrol-2020-055624).
\end{abstract}

${ }^{1}$ Behavioral Health and Health Policy Practice, Westat, Rockville, MD, USA

${ }^{2}$ Department of Health Behavior, Roswell Park Comprehensive Cancer Center, Buffalo, NY, United States

${ }^{3}$ Office of Science, Center for Tobacco Products, Food and Drug Administration, Silver Spring, MD, United States ${ }^{4}$ National Institute on Drug Abuse, National Institutes of Health, Bethesda, MD, United States

${ }^{5}$ Department of Oncology, Georgetown University Medical Center, Washington, DC, United States

\section{Correspondence to}

Dr Kathryn C Edwards, Behavioral Health and Health Policy, Westat, Rockville, MD 20852, USA;

KatyEdwards@westat.com

Received 13 January 2020 Revised 22 January 2020 Accepted 27 January 2020
Check for updates

(C) Author(s) (or their employer(s)) 2020. No commercial re-use. See rights and permissions. Published by BMJ.

To cite: Edwards $\mathrm{KC}$

Sharma E, Halenar MJ, et al. Tob Control

2020:29:s163-s169.

\section{ABSTRACT}

Objective The goal of this study is to examine the cross-sectional prevalence of use and 3-year longitudinal pathways of cigar use in US youth (12-17 years), young adults (18-24 years), and adults $25+$ (25 years or older). Design Data were drawn from the first three waves (2013-2016) of the Population Assessment of Tobacco and Health Study, a nationally representative, longitudinal cohort study of US youth and adults. Respondents with data at all three waves (youth, $n=11$ 046; young adults, $n=6478$; adults $25+, n=17$ 188) were included in longitudinal analyses.

Results Weighted cross-sectional prevalence of past 30-day (P30D) use was stable for adults 25+ ( 6\%), but decreased in youth (Wave $1(\mathrm{~W} 1)$ to Wave $3(\mathrm{~W} 3)=2.5 \%$ to $1.2 \%$ ) and young adults (W1 to $W 3=15.7 \%$ to $14.0 \%$ ). Among W1 P30D cigar users, over $50 \%$ discontinued cigar use (irrespective of other tobacco use) by Wave 2 (W2) or W3. Across age groups, over $70 \%$ of W1 P30D cigar users also indicated P30D use of another tobacco product, predominantly cigar polytobacco use with cigarettes. Discontinuing all tobacco use by W2 or W3 was greater in adult exclusive P30D cigar users compared with polytobacco cigar users.

Conclusions Although the majority of P30D cigar users discontinued use by W3, adult polytobacco users of cigars were less likely to discontinue all tobacco use than were exclusive cigar users. Tracking patterns of cigar use will allow further assessment of the population health impact of cigars.

\section{INTRODUCTION}

In the USA, cigar use peaked around 1950 and declined until the early 1990s but has risen dramatically since then. ${ }^{1}$ This is likely due to the introduction of products such as little cigars and cigarillos in addition to traditional large cigars. ${ }^{1-3}$ Although the patterns of use may differ by cigar type, the health risks (including various types of cancer and coronary heart disease) associated with cigar smoking are very similar to those of cigarette smoking. ${ }^{4}$ In Wave 1 (W1) of the Population Assessment of Tobacco and Health (PATH) Study (2013-2014), past 30-day (P30D) prevalence of cigar use was $2.5 \%$ for youth and 7.2\% for adults (specifically, P30D prevalence of traditional cigar, cigarillo and filtered cigar use was $0.7 \%, 2.2 \%$ and $0.5 \%$ among youth and 3.6\%, 4.4\% and $1.8 \%$ among adults, respectively). ${ }^{5}$ Prevalence of new past 12-month (P12M) cigar use from W1 to W3 (2013-2016) among US youth (9.0\%), young adults $(12.0 \%)$ and adults $25+(3.3 \%)$ indicates that more new use of cigar products occurs among young adults. ${ }^{6}$ Additional surveillance data (2012-2013) have shown that the majority (over 60\%) of cigar smokers indicate cigarillo use compared with other cigar types. ${ }^{7}$

In addition, over $10 \%$ of US adults were using multiple tobacco products in 2012-2013, and the combination of cigarettes and cigars was the highest multiple product use combination, with $2.6 \%$ of the population using these products. ${ }^{8}$ In cigar users, it is important to look at polytobacco use separately from exclusive cigar use since these users may use these products differently and have different health effects. ${ }^{8} 9$ Cigar polytobacco use also extends to youth. ${ }^{5}$ Research in adolescents has shown high levels of cigar, cigarillo and filtered cigar use among youth cigarette smokers, and this dual use is correlated with a number of negative health behaviours and outcomes. ${ }^{10}$ Dual cigar and cigarette use may be popular because little filtered cigars and cigarillos have physical characteristics similar to cigarettes but are less expensive and are available in a variety of flavours. ${ }^{11-14}$ Rostron et $a l^{15}$ showed that dual cigar and cigarette users were less likely to report intentions to quit all tobacco products than dual cigarette and e-cigarette users or polycigarette, cigar and e-cigarette users.

In 2016, the US Food and Drug Administration (FDA) extended its regulatory authority over the manufacture, distribution and marketing of tobacco products to include cigars, but currently cigar products do not face the same marketing and flavour restrictions as cigarettes. ${ }^{16} 17$ National, state and local tobacco control policies that seek to ban flavours in certain tobacco products could influence cigar use; however, this impact could differ depending on user characteristics (ie, age, exclusive vs polytobacco use). Understanding these patterns can potentially inform regulatory activities for cigars. 
This study broadens our understanding of cigar use by leveraging longitudinal data from three waves (2013-2016) of the PATH Study to examine pathways of cigar use in the USA. The first aim is to examine differences in cross-sectional weighted estimates of ever, P12M, P30D and daily P30D use for US youth (ages 12-17), young adults (ages 18-24) and adults $25+$ (ages 25 and older) for each of the first three waves. Drawing from longitudinal within-person data, the second aim is to examine age differences in W1-W2-W3 pathways of persistent use, discontinued use and reuptake of cigar use among W1 P30D cigar users. The final aim of this study is to compare longitudinal transitions of use among W1 exclusive cigar users, cigar polytobacco users with cigarettes (cigar polytobacco users w/CIGS) and cigar polytobacco users without cigarettes (cigar polytobacco users w/o CIGS) to understand pathways such as discontinued tobacco use, persistent cigar use and switching to another tobacco product. Examining transitions between use and nonuse of tobacco products separately for exclusive cigar and cigar polytobacco use will advance understanding of critical product transitions such as switching and complete tobacco cessation.

\section{METHODS}

\section{Study design and population}

The PATH Study is an ongoing, nationally representative, longitudinal cohort study of youth (ages 12-17) and adults (ages 18 or older) in the USA. Self-reported data were collected using audio computer-assisted self-interviews administered in English and Spanish. Further details regarding the PATH Study design and W1 methods are published elsewhere. ${ }^{18} 19$ At W1, the weighted response rate for the household screener was 54.0\%. Among screened households, the overall weighted response rate was $78.4 \%$ for youth and $74.0 \%$ for adults at W1, $87.3 \%$ for youth and $83.2 \%$ for adults at W2 and $83.3 \%$ for youth and $78.4 \%$ for adults at W3. Details on interview procedures, questionnaires, sampling, and weighting, and information on accessing the data are available on https://doi.org/10.3886/Series606. The study was conducted by Westat and approved by the Westat Institutional Review Board. All participants ages 18 and older provided informed consent, with youth participants ages 1217 providing assent while their parent/legal guardian provided consent.

The current study reports cross-sectional estimates from 13 651 youth and 32320 adults who participated in W1 (data collected 12 September 2013 through 14 December 2014), 12 172 youth and 28362 adults at W2 (23 October 2014 through 30 October 2015) and 11814 youth and 28148 adults at W3 (19 October 2015 to 23 October 2016). The differences in the number of completed interviews between W1, W2 and W3 reflect attrition due to non-response, mortality and other factors, as well as youth who enroll in the study at W2 or W3. ${ }^{18}$ We also report longitudinal estimates from W1 youth $(n=11$ $046), \mathrm{W} 1$ young adults $(\mathrm{n}=6478)$ and $\mathrm{W} 1$ adults $25+(\mathrm{n}=17$ 188 ) with data collected at all three waves. See online supplementary figure 1 for a detailed description of the analytic sample for longitudinal analysis.

\section{Measures}

\section{Tobacco use}

At each wave, adults and youth were asked about their tobacco use behaviours for cigarettes, electronic nicotine delivery systems (ENDS), traditional cigars, cigarillos, filtered cigars, pipe tobacco, hookah, snus pouches, other smokeless tobacco (loose snus, moist snuff, dip, spit or chewing tobacco) and dissolvable tobacco. Participants were asked about P30D use of 'e-cigarettes' at W1 and 'e-products' (e-cigarettes, e-cigars, e-pipes and e-hookah) at W2 and W3; for the purposes of this paper, all electronic products noted above are referred to as ENDS. In addition, youth were asked about their use of bidis and kreteks but these data were not included in the analyses due to small sample sizes.

Pictures, descriptions and common brands were displayed for each product (except cigarettes) prior to questioning. A description of how cigar use was defined in the PATH Study was published previously. ${ }^{20}$ Briefly, respondents were presented with images and common brands of traditional cigars. After answering questions about their traditional cigar use, images of cigarillos and filtered cigars were shown, and the products were described as 'smaller than traditional cigars. They are usually brown. Some are the same size as cigarettes, and some come with tips or filters.' Follow-up questions differentiated filtered cigars from cigarillos by asking respondents if they smoked a product 'with a filter (like a cigarette filter)' (which would indicate filtered cigars) or a product 'with a plastic or wooden tip' or 'without a tip or filter' (cigarillos). Due to low sample sizes, responses were combined to represent any cigar use, such that if a respondent indicated using at least one of the products described above, they were considered a cigar user.

\section{Outcome measures}

Cross-sectional definitions of use included ever, P12M, P30D and daily P30D use. Longitudinal outcomes included persistent cigar use (continued exclusive or polytobacco cigar use at W2 and W3), discontinued cigar use (stopped cigar use at W2 and W3 or just W3) and reuptake of cigar use (used cigars at W1, discontinued cigar use at W2 and used cigars again at W3), as well as transitions among exclusive and polytobacco cigar users. The definition of each outcome is included in the footnote of the table/figure in which it is presented.

\section{Analytic approach}

To address Aim 1, weighted cross-sectional prevalence of cigar use was compared across waves for each age group using various definitions of use (eg, ever, P12M, P30D, daily P30D). Crosssectional estimates of individual cigar product use or multiple cigar product use at each wave (stratified by age) were also examined. For Aim 2, irrespective of other tobacco product use, longitudinal W1-W2-W3 transitions in P30D cigar use were compared by age group. The pathways of interest for Aim 2 include persistent any P30D cigar use, discontinued any P30D cigar use and reuptake of any P30D cigar use. Finally, for Aim 3, longitudinal W1-W2-W3 cigar use pathways that flow through seven mutually exclusive and exhaustive transition categories were examined for W1 P30D exclusive cigar use, W1 P30D cigar polytobacco use w/CIGS and W1 P30D cigar polytobacco use w/o CIGS (see online supplementary figure 2). For each aim, weighted t-tests were conducted on differences in proportions to assess statistical significance. To correct for multiple comparisons, Bonferroni post hoc tests were conducted. Given that cigarettes are the most commonly used tobacco product with the most robust evidence base of potentially harmful health consequences, ${ }^{1}$ two polytobacco use groups were examined separately to compare longitudinal transitions among polytobacco users who use and do not use cigarettes. These pathways represent building blocks that may be aggregated to reflect higher-level behavioural transitions.

Cross-sectional estimates (Aim 1) were calculated using PATH Study cross-sectional weights for W1 and singlewave (pseudo-cross-sectional) weights for W2 and W3. The 


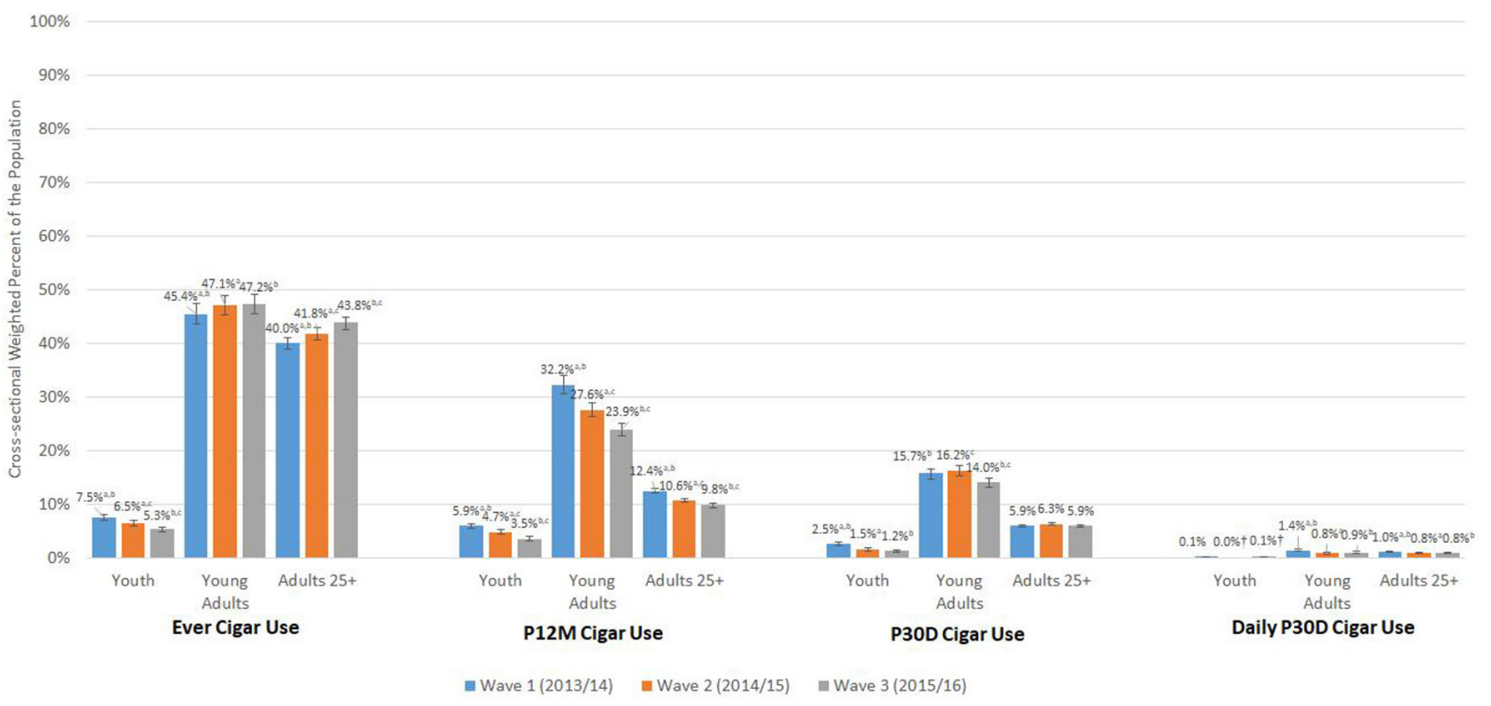

Figure 1 Cross-sectional weighted percent of ever, P12M, P30D and daily P30D cigar use among youth, young adults and adults 25+ in W1, W2 and W3 of the Population Assessment of Tobacco and Health (PATH) Study.

Notes: Abbreviations: P12M = past 12-month; P30D = past 30-day; W1 = Wave 1; W2 = Wave 2; W3 = Wave 3

W1/W2/W3 ever cigar use unweighted Ns:youth (ages 12-17) = 998/765/586; young adults (ages 18-24) = 5,092/4,328/4,109; adults 25+ (ages 25 and older) $=12,128 / 11,116 / 11,174$

W1/W2/W3 P12M cigar use unweighted Ns: youth = 791/570/394; young adult =3,725/2,665/2,250; adults 25+=5,179/3,892/3,430

W1/W2/W3 P30D cigar use unweighted Ns: youth = 340/180/136; young adult =1,933/1,649/1,379; adults 25+ =2,640/2,401/2,207

W1/W2/W3 daily P30D cigar use unweighted Ns: youth = 11/4/8; young adults $=202 / 92 / 97$; adults $25+=485 / 338 / 331$

X-axis shows four categories of cigar use (ever, P12M, P30D, and daily P30D). Y-axis shows weighted percentages of W1,W2, and W3 users. Sample analyzed includes all W1, W2, and W3 respondents at each wave. All respondents with data at one wave are included in the sample for that wave's estimate and do not need to have complete data at all three waves. The PATH Study cross-sectional (W1) or single-wave weights (W2 and W3) were used to calculate estimates at each wave. Ever cigar use is defined as having used a traditional cigar, cigarillo, and/or filtered cigar, even once or twice in lifetime. P12M cigar use is defined as any traditional cigar, cigarillo, and/or filtered cigar use within the past 12-months. P30D cigar use is defined as any traditional cigar, cigarillo, and/or filtered cigar use within the past 30 days. Daily P30D cigar use is defined as use of traditional cigars, cigarillos, and/or filtered cigars on all 30 of the past 30 days. All use definitions refer to any use that includes exclusive or polytobacco use of cigars.

a denotes significant difference at $p<0.0167$ (Bonferroni corrected for three comparisons) between W1 and W2

bdenotes significant difference at $p<0.0167$ (Bonferroni corrected for three comparisons) between W1 and W3

'denotes significant difference at $p<0.0167$ (Bonferroni corrected for three comparisons) between W2 and W3

The logit-transformation method was used tocalculate the $95 \%$ confidence intervals.

†Estimate should be interpreted with caution because it has low statistical precision. It is based on a denominator sample size of less than 50 , or the coefficient of variation of the estimate or its complement is larger than $30 \%$.

Analyses were run on the W1, W2, and W3 Public Use Files (https://doi.org/10.3886/ICPSR36498.v8).

weighting procedures adjusted for complex study design characteristics and non-response. Combined with the use of a probability sample, the weighted data allow these estimates to be representative of the non-institutionalised, civilian, resident US population aged 12 or older at the time of each wave. Longitudinal estimates (Aims 2 and 3) were calculated using the PATH Study W3 all-waves weights. These weighted estimates are representative of the resident US population aged 12 and older at the time of W3 (other than those who were incarcerated) who were in the civilian, non-institutionalised population at W1.

All analyses were conducted using SAS Survey Procedures V.9.4 (SAS Institute). Variances were estimated using the balanced repeated replication method ${ }^{21}$ with Fay's adjustment set to 0.3 to increase estimate stability. ${ }^{22}$ Analyses were run on the W1-W3 Public Use Files (https://doi.org/10.3886/ ICPSR36498.v8). Estimates with low precision (fewer than 50 observations in the denominator or with a relative standard error greater than 0.30 ) were flagged and are not discussed in the Results section.

\section{RESULTS}

\section{Cross-sectional weighted prevalence}

As shown in figure 1, P12M use decreased from W1 to W3 in all age groups. Daily cigar use was almost non-existent among youth $(\leq 0.1 \%$ at each wave), and minimal in young adults $(\leq 1.4 \%$ at each wave) and adults $25+(\leq 1.0 \%$ at each wave).

Prevalence of P30D use was relatively stable in adults $25+$, yet declined in young adults (W2: $16.2 \%$ (95\% CI 15.3 to 17.1 ); W3: $14.0 \%$ (95\% CI 13.1 to 14.9$)$ ) and youth (W1: 2.5\% (95\% CI 2.2 to 2.8 ); W3: $1.2 \%$ (95\% CI 1.0 to 1.4$)$ ). As shown in online supplementary table 1 , among the $1.2 \%$ of youth P30D cigar users at W3, 50.4\% (95\% CI 40.4 to 60.3) used cigarillos exclusively and $23.6 \%$ (95\% CI 17.2 to 31.5 ) were polycigar users. Among the $14.0 \%$ of young adult P30D cigar users at W3, $44.8 \%$ (95\% CI 41.9 to 47.8 ) used cigarillos only, and $34.9 \%$ (95\% CI 31.9 to 38.0 ) were polycigar users. Among the $5.9 \%$ of adult 25 + P30D cigar users at W3, 28.8\% (95\% CI 26.2 to 31.5) used traditional cigars only, 23.8\% (95\% CI 21.8 to 26.0$)$ used cigarillos only, $12.4 \%$ (95\% CI 10.6 to 14.3 ) used filtered cigars only and $35.0 \%$ (95\% CI 32.4 to 37.8 ) were polycigar users. 


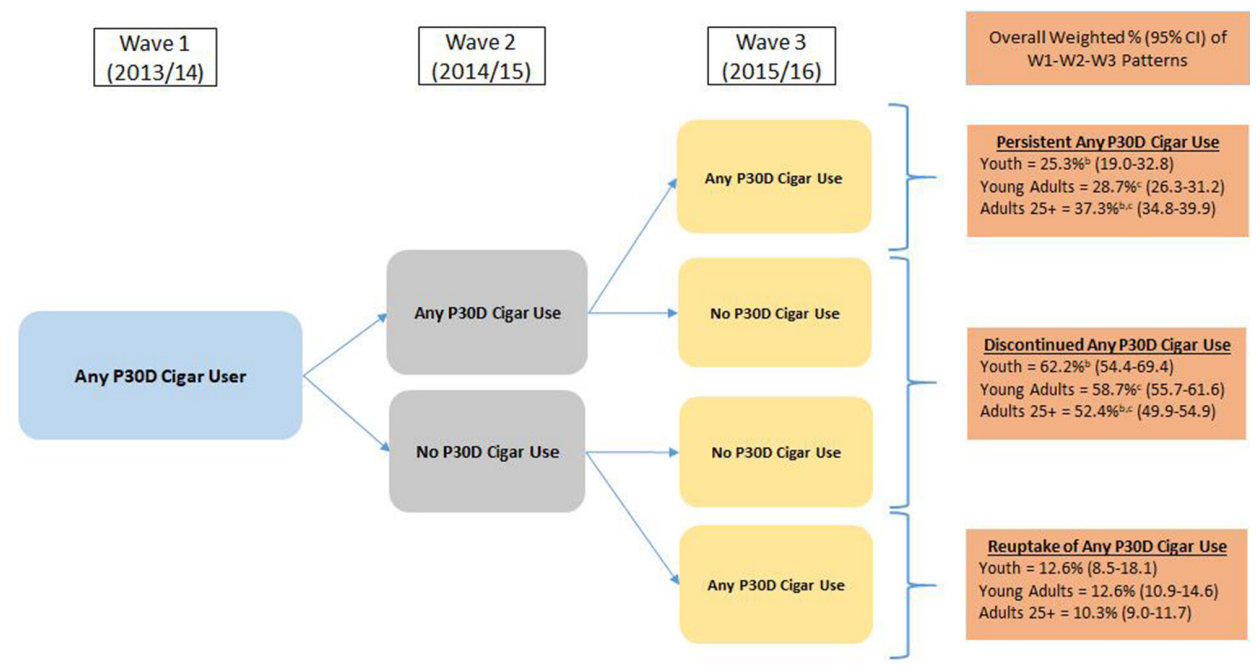

Figure 2 Patterns of W1-W2-W3 persistent any P30D cigar use, discontinued any P30D cigar use and reuptake of any P30D cigar use among W1 any P30D cigar users.

Notes: Abbreviations: $\mathrm{W} 1=$ Wave $1 ; \mathrm{W} 2=$ Wave 2; $\mathrm{W} 3=$ Wave 3; P30D = past 30-day; $\mathrm{Cl}=$ confidence interval

W1 any P30D cigar use weighted percentages $(95 \% \mathrm{Cl})$ out of total U.S. population: youth (ages 12-17) = 2.5\% (2.2-2.9); young adults (ages 18-24) = $15.6 \%$ (14.6-16.7); adults 25+ (ages 25 and older) $=5.9 \%(5.5-6.2)$

Analysis included W1 youth, young adults, and adults 25+ P30D cigar users with data at all three waves. Respondent age was calculated based on age at W1. W3 longitudinal (all-waves) weights were used to calculate estimates. These rates vary slightly from those reported in Figure 1 or Supplemental Table 1 because this analytic sample in Figure 2 includes only those with data at each of the three waves to examine weighted longitudinal use and non-use pathways.

Any P30D cigar use was defined as any use of traditional cigars, cigarillos, and/or filtered cigars within the past 30 days. Respondent could be missing data on other P30D tobacco product use and still be categorized into the following three groups:

1) Persistent any P30D cigar use: Defined as exclusive or cigar polytobacco use at W2 and W3.

2) Discontinued any P30D cigar use: Defined as any non-cigar tobacco use or no tobacco use at either W2 and W3 or just W3.

3) Reuptake of any P30D cigar use: Defined as discontinued any cigar use at W2 and any cigar use at W3.

a denotes significant difference at $p<0.0167$ (Bonferroni corrected for three comparisons) between youth and young adults

bdenotes significant difference at $p<0.0167$ (Bonferroni corrected for three comparisons) between youth and adults 25+

'denotes significant difference at $p<0.0167$ (Bonferroni corrected for three comparisons) between young adults and adults $25+$

The logit-transformation method was used to calculate the $95 \% \mathrm{Cls}$.

Analyses were run on the W1, W2, and W3 Public Use Files (https://doi.org/10.3886/ICPSR36498.v8).

\section{Longitudinal weighted W1-W2-W3 pathways}

\section{Among any P30D cigar users at W1}

To address Aim 2, we examined pathways of persistent use, discontinued use and reuptake of use among the $2.5 \%$ (95\% CI 2.2 to $2.9)$ of youth, $15.6 \%$ (95\% CI 14.6 to 16.7$)$ of young adults and $5.9 \%(95 \%$ CI 5.5 to 6.2 ) of adults $25+$ who had used any cigar in the past 30 days at $\mathrm{W} 1$. The relative proportion of persistent any P30D cigar use at all three waves, irrespective of concurrent other product use, was $25.3 \%$ (95\% CI 19.0 to 32.8 ) among youth, $28.7 \%$ (95\% CI 26.3 to 31.2$)$ among young adults and greatest among adults $25+(37.3 \%$ (95\% CI 34.8 to 39.9$))$. A larger relative proportion of youth (62.2\% (95\% CI 54.4 to 69.4$))$ and young adults (58.7\% (95\% CI 55.7 to 61.6)) discontinued P30D cigar use either at W2 or W3 than adults $25+(52.4 \%$ (95\% CI 49.9 to 54.9)). Lastly, there were no significant differences by age group for relative proportion of cigar reuptake, defined as W1 cigar users who had no P30D cigar use at W2 and went back to P30D cigar use again at W3 (youth: $12.6 \%$ (95\% CI 8.5 to 18.1 ); young adults: $12.6 \%$ (95\% CI 10.9 to 14.6$)$; and adults $25+: 10.3 \%$ (95\% CI 9.0 to 11.7$)$ ) (figure 2 ).

Among P30D exclusive cigar users, P30D cigar polytobacco users w/ CIGS and P30D cigar polytobacco users w/o CIGS at W1

Most of the W1 P30D cigar users also used another tobacco product in the past 30 days (youth, $76.7 \%$ (95\% CI 70.9 to 81.6); young adults, $81.3 \%$ (95\% CI 79.0 to 83.4 ); adults $25+, 71.1 \%$ (95\% CI 68.0 to 73.9 ); results not shown). A total of $55.0 \%$ (95\% CI 47.6 to 62.3 ) of youth, $68.0 \%$ (95\% CI 64.8 to 71.0 ) of young adults and $63.9 \%$ (95\% CI 60.9 to 66.9$)$ of adults $25+$ were cigar polytobacco users w/CIGS, and among those, 39.1\% (95\% CI 31.1 to 47.8 ) of youth, $38.5 \%$ (95\% CI 35.2 to 41.9$)$ of young adults and $56.8 \%$ (95\% CI 53.9 to 59.6) of adults $25+$ were dual cigar and cigarette users (results not shown).

For Aim 3, pathways were examined across seven mutually exclusive categories (see conceptual map in online supplementary figure 2) among three separate W1 groups who used any cigar products in the past 30 days: (1) P30D exclusive cigar users (online supplementary table 2a), (2) P30D cigar polytobacco users w/CIGS (online supplementary table 2b), and (3) P30D cigar polytobacco users w/o CIGS (online supplementary table 2c). Described next are summary pathways shown in table 1 that estimate broad behavioural transitions such as persistent use and tobacco cessation.

Among youth (table 1). 35.5\% (95\% CI 23.1 to 50.3) of exclusive users, and $20.3 \%$ (95\% CI 14.4 to 27.9 ) of cigar polytobacco w/CIGS users discontinued all tobacco use by W3. Persistent use was greater in cigar polytobacco w/CIGS users $(24.7 \%(95 \%$ CI 17.0 to 34.4 )) than exclusive or cigar polytobacco users w/o CIGS (less than $6 \%$ across these groups). Switching to use of other tobacco product(s) by W3 occurred in 20.0\% (95\% CI 


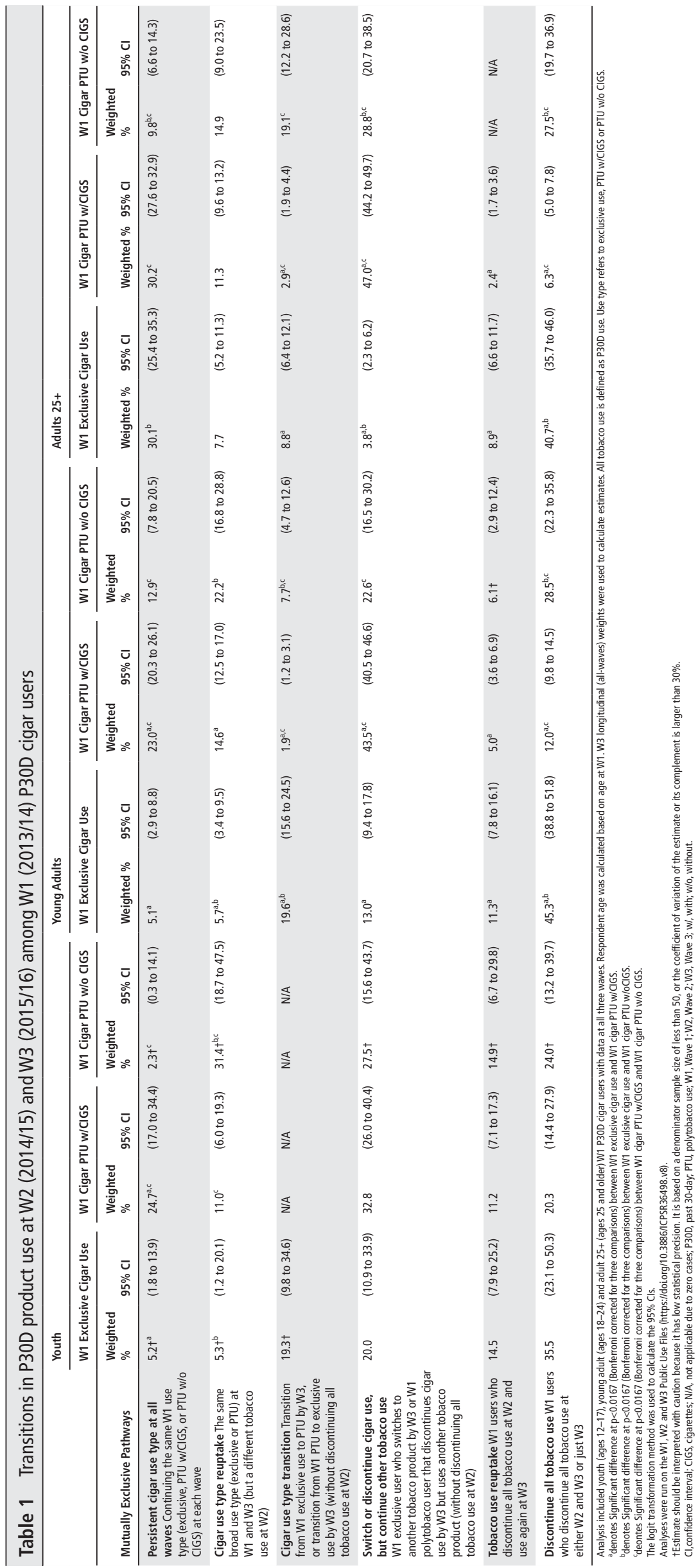


10.9 to 33.9 ) of exclusive users, and $32.8 \%$ (95\% CI 26.0 to 40.4) cigar polytobacco w/CIGS users.

Among young adults (table 1). Discontinuation of all tobacco use was larger for exclusive users (45.3\% (95\% CI 38.8 to 51.8$)$ ) than cigar polytobacco users w/o CIGS (28.5\% (95\% CI 22.3 to $35.8)$ ), which was larger than cigar polytobacco users w/CIGS (12.0\% (95\% CI 9.8 to 14.5$)$ ). Roughly a quarter maintained persistent cigar polytobacco use w/CIGS over three waves, $23.0 \%$ (95\% CI 20.3 to 26.1), which was greater than persistent exclusive use (5.1\% (95\% CI 2.9 to 8.8$)$ ) and persistent polytobacco w/o CIGS (12.9\% (95\% CI 7.8 to 20.5$)$ ). Transitioning from exclusive to polytobacco use by W3 occurred in $19.6 \%$ (95\% CI 15.6 to 24.5 ) of young adults, which was greater than the percentage who transitioned from polytobacco cigar use to exclusive use (w/ CIGS: $1.9 \%$ (95\% CI 1.2 to 3.1); w/o CIGS: 7.7\% (95\% CI 4.7 to 12.6)). A larger proportion of W1 cigar polytobacco users w/ CIGS, 43.5\% (95\% CI 40.5 to 46.6), discontinued cigar use by W3 but continued other tobacco use compared with exclusive or polytobacco w/o CIGS users (range: 13\%-23\%).

Among adults $25+$ (table 1$)$. Similar to young adults, over $40 \%$ of exclusive cigar users discontinued all tobacco use by W3 compared with roughly a quarter $(27.5 \%$ (95\% CI 19.7 to $36.9)$ ) of cigar polytobacco users w/o CIGS and only $6.3 \%(95 \%$ CI 5.0 to 7.8 ) of cigar polytobacco users w/CIGS. Persistent use was similar $(\sim 30.0 \%)$ between exclusive and cigar polytobacco use w/CIGS users, but was seen in only 9.8\% (95\% CI 6.6 to 14.3) of cigar polytobacco users w/o CIGS. Switching to other tobacco product use only occurred in $3.8 \%$ (95\% CI 2.3 to 6.2 ) of exclusive users, but discontinuing cigar and continuing other tobacco use represented $47.0 \%$ (95\% CI 44.2 to 49.7 ) of cigar polytobacco users w/CIGS and $28.8 \%$ (95\% CI 20.7 to 38.5 ) of cigar polytobacco users w/o CIGS.

\section{DISCUSSION}

The current study found decreasing prevalence of cigar use in the USA from 2013 to 2016 when assessing P12M use across age groups, P30D use for youth and young adults, as well as negligible $(<1.5 \%)$ daily use across age groups. Exclusive cigarillo use was among the most common patterns of use among cigar users (past 30 days, past 12 months) particularly for youth and young adults and less so for adults $25+$. These cross-sectional results are fairly consistent with those in the literature, ${ }^{5}$ although the rates of use in youth in the PATH Study are lower than those reported by the National Youth Tobacco Survey 2016. ${ }^{23}$ This may be due to different age groups included in each estimate (eg, 18 year-olds are considered to be adults in the PATH Study, even if they are still in high school), skip patterns in the data collection instruments and findings that household-based assessments of tobacco use tend to result in lower prevalence rates than do school-based assessments. ${ }^{24}$ Recent P30D cigar use estimates (an average of $7.6 \%$ for all adults, $\mathrm{W} 1)^{5}$ were greater than estimates from 10 years ago $(5.5 \%$ among all adults in 2003$),{ }^{2}$ when cigar consumption was increasing. ${ }^{1}$

Longitudinal patterns of use over a 3-year period revealed that the most common pathway of cigar use among youth, young adults and adults $25+$ was discontinuing P30D cigar use at W2 or W3 (irrespective of other tobacco product use), capturing over half of P30D cigar users. Only about a quarter to a third of W1 P30D cigar users continued P30D cigar use at W2 and W3. Among youth and all adults, over 70\% of W1 P30D cigar users were polytobacco users, with over 55\% also using cigarettes; of those, over $38 \%$ were dual cigar and cigarette users. This finding supports previous reports of multiple tobacco product use, specifically the high proportion of dual cigar and cigarette use. $^{8}{ }^{10-13}$ Regardless of exclusive or polytobacco use status at W1, longitudinal pathways of P30D cigar use over 3 years show that discontinued P30D use of cigars (or all tobacco products) was the most common pathway. However, since these analyses show that cigars may be used intermittently (eg, non-daily) across all age groups, additional waves of data can determine if this discontinued use is reflective of long-term cessation. Patterns of transitions for cigar polytobacco use with and without cigarette use differed across the three waves. For cigar polytobacco use w/CIGS, discontinuing cigar use but maintaining other tobacco use was one of the most common transitions across age groups. Adult cigar polytobacco users w/o CIGS were just as likely to discontinue all tobacco use as to discontinue cigar use but maintain other tobacco use.

Among adults, over one-third of exclusive cigar users and exclusive ENDS users discontinued all tobacco use, while among youth, $\sim 54 \%$ of exclusive ENDS users compared with $\sim 36 \%$ of exclusive cigar users discontinued all tobacco use. ${ }^{25}$ Discontinuing all tobacco was less common $(<25 \%)$ across age groups for smokeless tobacco and cigarette users, regardless of exclusive or multiple product use. ${ }^{2627}$ When compared with other polytobacco users, across age groups, over a third of W1 cigar polytobacco users w/CIGS discontinued cigar use but continued other tobacco use at W3, similar to patterns seen in polytobacco users of ENDS with cigarettes. ${ }^{25}$ In addition, over $20 \%$ of young adult W1 cigar polytobacco users w/o CIGS discontinued cigar use but continued other product use similar to young adult hookah polytobacco use w/o CIGS and young adult smokeless tobacco polytobacco use w/o CIGS. ${ }^{28} 29$

\section{Limitations}

Although we presented cross-sectional estimates of individual cigar product use among cigar users, we were limited in our ability to assess longitudinal pathways by individual product use due to small sample sizes. Other limitations include the possibility of recall bias from self-report measures, although measures were taken to accurately describe which products respondents were asked about (see the Methods section). Weighted longitudinal analyses over the 3-year period excluded participants who were missing data at one of the waves (2605 youth and 8650 adults). The extent of missing data and the small number of observations for specific low-prevalence pathways may limit interpretation. However, this report is a resource that provides building blocks to aggregate different pathways to explore a variety of research questions regarding cigar use. Future studies are needed to examine adjusted models and to determine which factors predict priority pathways and drive different patterns of use. Kasza et $a l^{3031}$ and Edwards et $a l^{32}$ examine demographic correlates of initiation, cessation and relapse of cigar and other tobacco product use to further explore predictors of these critical outcomes.

\section{Summary and implications}

The FDA has issued advance notice of proposed rulemakings to seek input on the impact of flavours in tobacco products and lowering nicotine in cigarettes to minimal or non-addictive levels. Future regulations in these areas would likely impact patterns of use for cigars. Given that cigarette and cigar polytobacco use is common, and that the use of cigarettes along with cigars appears to hamper discontinuation of all tobacco products, continuing to track these patterns of use will allow for more robust evaluations of the health risks of cigar products. 
What this paper adds

- Cross-sectional prevalence of past 30-day (P30D) cigar use was relatively stable in adults $25+$ but decreased in youth and young adults over 3 years.

- Over 70\% of Wave 1 (W1) P30D cigar users also used another tobacco product. The majority of cigar users were polytobacco users who use cigarettes.

- Discontinuing all tobacco use by W2 or W3 was greater in adult exclusive P30D cigar users compared with polytobacco cigar users.

- Adult cigar polytobacco users without cigarettes were more likely to discontinue all tobacco than those who are cigar polytobacco users with cigarettes.

Contributors KCE and CAS led the conceptual design. KCE drafted the initial manuscript and all authors critically revised it. ES and MJH conducted the statistical analysis and all authors contributed to interpretation of results. All authors approved the work for journal publication and agree to be accountable for all aspects of the work.

Funding This manuscript is supported with federal funds from the National Institute on Drug Abuse, National Institutes of Health, and the Center for Tobacco Products, Food and Drug Administration, Department of Health and Human Services, under a contract to Westat (contract number HHSN271201100027C).

Disclaimer The findings and conclusions in this report are those of the authors and do not necessarily represent the official position of the US Department of Health and Human Services or any of its affiliated institutions or agencies.

Competing interests WMC reports long-term stock holdings in General Electric Company, 3M Company, and Pfizer Incorporated, unrelated to this manuscript. No financial disclosures were reported by the other authors of this paper.

Patient consent for publication Not required.

Ethics approval The study was conducted by Westat and approved by the Westat Institutional Review Board. All participants aged 18 and older provided informed consent, with youth participants aged 12-17 providing assent while their parent/ legal guardian provided consent.

Provenance and peer review Not commissioned; internally peer reviewed.

Data availability statement Data from the PATH Study Wave 1 to Wave 3 are available for download as Public Use Files in a public, open access repository (https://www.icpsr.umich.edu/icpsrweb/NAHDAP/studies/36498). Conditions of use are available at the website above.

\section{ORCID iDs}

Kathryn C Edwards http://orcid.org/0000-0002-0645-1705

Eva Sharma http://orcid.org/0000-0002-1328-508X

Michael J Halenar http://orcid.org/0000-0002-8703-3811

Kristie A Taylor http://orcid.org/0000-0001-6668-7923

Karin A Kasza http://orcid.org/0000-0002-4492-098X

Maansi Bansal-Travers http://orcid.org/0000-0002-7944-3570

Lisa D Gardner http://orcid.org/0000-0003-3340-2818

Heather L Kimmel http://orcid.org/0000-0001-8278-0095

Cassandra A Stanton http://orcid.org/0000-0001-5329-6261

\section{REFERENCES}

1 US Department of Health and Human Services. The health consequences of Smoking — 50 years of progress: a report of the surgeon General. 943. Atlanta, GA: US Department of Health and Human Services, Centers for Disease Control and Prevention, National Center for Chronic Disease Prevention and Health Promotion, Office on Smoking and Health, 2014.

2 Delnevo CD. Smokers' choice: what explains the steady growth of cigar use in the U.S.? Public Health Rep 2006:121:116-9.

3 Center for Disease Control and Prevention. CDC Fact Sheet - Cigars, 2016. Available: https://www.cdc.gov/tobacco/data_statistics/fact_sheets/tobacco_industry/cigars/ index.htm [Accessed 26 Feb 2017].

4 Chang CM, Corey CG, Rostron BL, et al. Systematic review of cigar smoking and all cause and smoking related mortality. BMC Public Health 2015;15:390.

5 Kasza KA, Ambrose BK, Conway KP, et al. Tobacco-Product use by adults and youths in the United States in 2013 and 2014. N Eng/ J Med Overseas Ed 2017:376:342-53.
6 Stanton CA, Sharma E, Seaman EL, et al. Initiation of any tobacco and five tobacco products across 3 years among youth, young adults, and adults in the United States: findings from the path study waves 1-3 (2013-2016). Tob Control 2020;29:s178-90.

7 Corey C, King B, Coleman B, et al. Little filtered cigar, cigarillo, and premium cigar smoking among adults-United states, 2012-2013. MMWR Morb Mortal Wkly Rep 2014;63:650.

8 Lee YO, Hebert CJ, Nonnemaker JM, et al. Multiple tobacco product use among adults in the United States: cigarettes, cigars, electronic cigarettes, hookah, smokeless tobacco, and snus. Prev Med 2014;62:14-19.

9 Pickworth WB, Rosenberry ZR, O'Grady KE, et al. Dual use of cigarettes, little cigars, cigarillos, and large cigars: smoking topography and toxicant exposure. Tobacco Regulatory Science 2017;3:72-83.

10 Schuster RM, Hertel AW, Mermelstein R. Cigar, cigarillo, and little cigar use among current cigarette-smoking adolescents. Nicotine Tob Res 2013;15:925-31.

11 Sterling K, Berg CJ, Thomas AN, et al. Factors associated with small cigar use among college students. Am J Health Behav 2013;37:325-33.

12 Portnoy DB, Wu CC, Tworek C, et al. Youth curiosity about cigarettes, smokeless tobacco, and cigars: prevalence and associations with advertising. Am J Prev Med 2014:47:S76-86

13 Cohn A, Cobb CO, Niaura RS, et al. The other Combustible products: prevalence and correlates of little Cigar/Cigarillo use among cigarette smokers. NICTOB 2015;17:1473-81.

14 Kong G, Bold KW, Simon P, et al. Reasons for Cigarillo initiation and Cigarillo manipulation methods among adolescents. Tob Regul Sci 2017;3:48-58.

15 Rostron BL, Schroeder MJ, Ambrose BK. Dependence symptoms and cessation intentions among US adult daily cigarette, cigar, and e-cigarette users, 2012-2013. BMC Public Health 2016;16:814.

16 Family smoking prevention and tobacco control act. Public Law 2009:111-31.

17 Food and Drug Administration (HHS). Deeming tobacco products to be subject to the federal food, drug, and cosmetic act, as amended by the family smoking prevention and tobacco control act; restrictions on the sale and distribution of tobacco products and required warning statements for tobacco products. final rule. Fed Regist 2016;81:28973-9106.

18 Hyland A, Ambrose BK, Conway KP, et al. Design and methods of the population assessment of tobacco and health (path) study. Tob Control 2017;26:371-8.

19 Tourangeau R, Yan T, Sun H, et al. Population assessment of tobacco and health (path) reliability and validity study: selected reliability and validity estimates. Tob Control 2019;28:663-8.

20 Corey CG, Holder-Hayes E, Nguyen AB, et al. Us adult cigar smoking patterns, purchasing behaviors, and reasons for use according to cigar type: findings from the population assessment of tobacco and health (path) study, 2013-2014. Nicotine \& Tobacco Research 2017

21 McCarthy PJ. Pseudoreplication: further evaluation and applications of the balanced half-sample technique 1969.

22 Judkins DR. Fay's method for variance estimation. Journal of Official Statistics 1990:6:223

23 Jamal A, Gentzke A, Hu SS, et al. Tobacco Use Among Middle and High School Students - United States, 2011-2016. MMWR Morb Mortal Wkly Rep 2017;66:597-603.

24 Brener ND, Billy JOG, Grady WR. Assessment of factors affecting the validity of self-reported health-risk behavior among adolescents: evidence from the scientific literature. J Adolesc Health 2003:33:436-57.

25 Stanton CA, Sharma E, Edwards KC, et al. Longitudinal transitions of exclusive and polytobacco electronic nicotine delivery systems (ends) use among youth, young adults, and adults in the USA: findings from the PATH Study Waves 1-3 (2013-2016). Tob Control 2020;29:5147-54.

26 Sharma E, Edwards KC, Halenar MJ, et al. Longitudinal pathways of exclusive and polytobacco smokeless use among youth, young adults, and adults in the USA: findings from the PATH Study Waves 1-3 (2013-2016). Tob Control 2020;29:s170-7.

27 Taylor KA, Sharma E, Edwards KC, et al. Longitudinal pathways of exclusive and polytobacco cigarette use among youth, young adults, and adults in the USA: findings from the PATH Study Waves 1-3 (2013-2016). Tob Control 2020;29:5139-46.

28 Edwards KC, Sharma E, Halenar MJ, et al. Longitudinal pathways of exclusive and polytobacco cigar use among youth, young adults, and adults in the USA: findings from the PATH Study Waves 1-3 (2013-2016). Tob Control 2020;29:s163-9.

29 Sharma E, Bansal-Travers M, Edwards KC, et al. Longitudinal pathways of exclusive and polytobacco hookah use among youth, young adults, and adults in the USA: findings from the PATH Study Waves 1-3 (2013-2016). Tob Control 2020:29:s155-62.

30 Kasza K, Edwards KC, Tang Z, et al. Correlates of tobacco product initiation among youth and adults in the USA: findings from the PATH Study Waves 1-3 (2013-2016). Tob Control 2020;29:5191-202.

31 Kasza K, Edwards KC, Tang Z, et al. Correlates of tobacco product cessation among youth and adults in the USA: findings from the PATH Study Waves 1-3 (2013-2016). Tob Control 2020;29:s203-15.

32 Edwards KC, Kasza K, Tang Z, et al. Correlates of tobacco product relapse among youth and adults in the USA: findings from the PATH Study Waves 1-3 (2013-2016). Tob Control 2020;29:s216-26. 